\title{
18. DISCUSSION FOLLOWING LÜST'S REPORT
}

(Sunday, September 14, 1969)

\author{
Chairman: R. WEYMANN
}

Editor's remark: This discussion actually took place after the Reports by Lüst and by Pottasch. However, the remarks pertaining to Pottasch's Report have been combined with the discussion following Boyarchuk's Report, since the latter two Reports appeared to have more in common than the first two. I have condensed several contributions, notably a very long remark by Gordon.

Severnyi: I would like to discuss a comparison of Crimean measurements of the average solar magnetic field with direct measurements of interplanetary magnetic fields made with the aid of Explorer 33 and 35 (Wilcox et al., 1969). Let me mention first that we have been measuring the average solar magnetic field since 1967 . We use the parallel beam of solar light passing through the entrance slit of the solar magnetograph. So we are recording the average magnetic field of the Sun, as if it were a star, where the weighting function is the distribution of brightness over the disk. Figure 1 shows the results. In the upper half we plot as a function of time the average solar field and the results on the interplanetary fields by the Explorers (a small time delay is taken into account). You see that there is an almost complete coincidence of sign between the general magnetic field and the interplanetary field. Even very small peaks in the general field, which I did not think had a real meaning, correspond closely to the reversals obtained by the Explorers. I want to stress that the general magnetic field is essentially a background field and not the resultant field of all the sunspots together. Indeed, in the lower half of Figure 1 the total sunspot field is shown. It clearly is in antiphase with the general magnetic field. This probably means that there is some tendency to compensate the excess of magnetism of background field. Now I wish to stress that such a close correspondence between the solar magnetic field and the interplanetary field can arise only if the field on the disk is ordered on a large scale. This supposition is supported by results obtained a couple of years ago by Wilcox for the solar wind. (Wilcox, J. M., Severnyi, A., and Colburn, D. S. : 1969, Nature 224, 353.)

Meyer: I would like to add to this picture some recent results obtained by Howard at Mt. Wilson Observatory, concerning large-scale velocity structures on the solar surface. With high Doppler resolution Howard observed apparently systematic upward and downward velocities appearing in alternating areas covering the Sun in about the same way that these magnetic sectors do. It will be very interesting to learn about a possible correlation between the two phenomena and their relation to the hypothetical 'giant granulation cells'. Structures of a similar kind are also indicated by recent hydrodynamic investigations by Busse dealing with the convection zone patterns in rotating stars and in the Sun. 


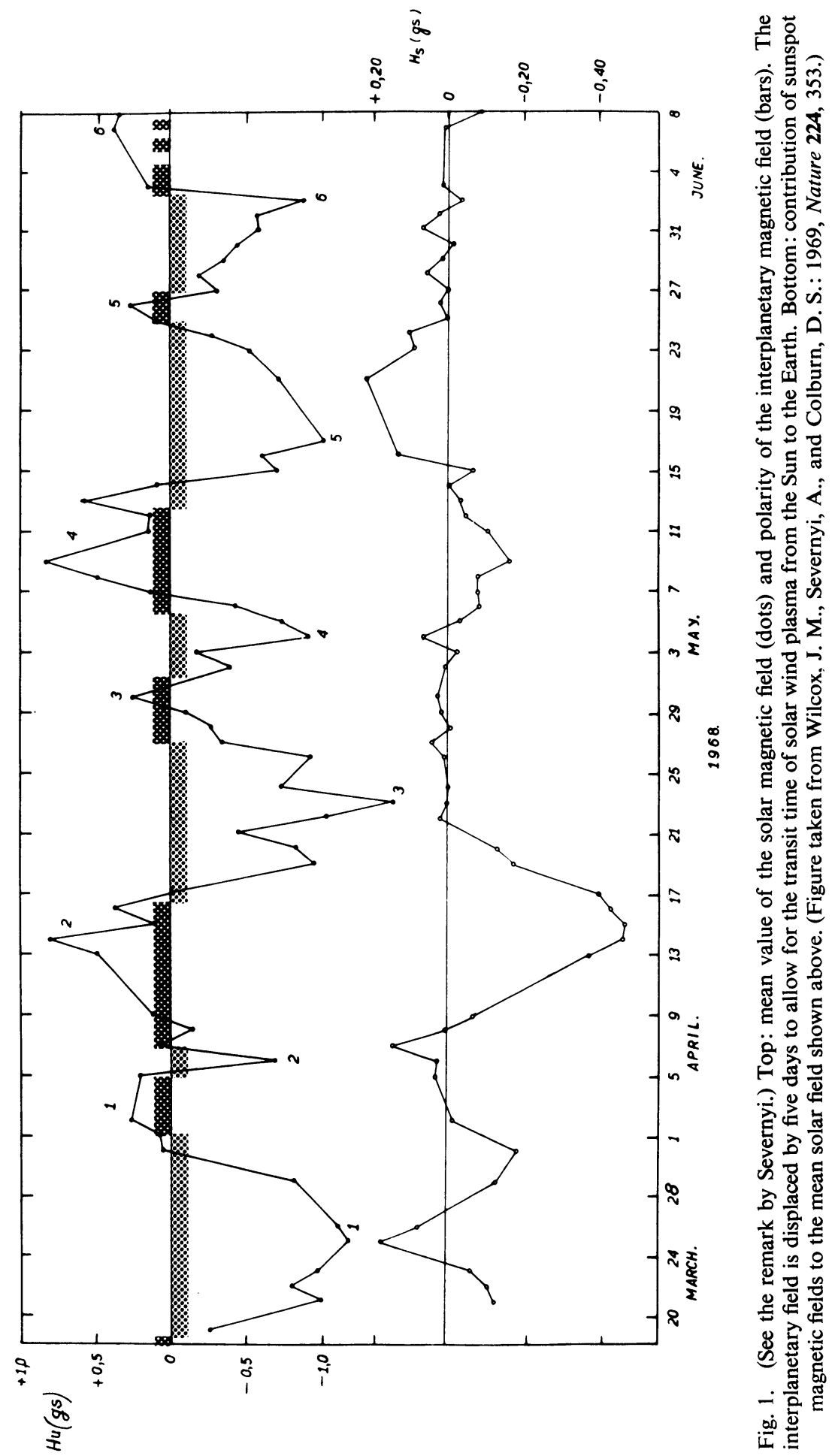


Burke: Dr. Severnyi, to get a good agreement what time delay was put in between the solar measurements and the satellite data?

Severnyi: A time delay of 4.2 days.

Mestel: A question to Dr. Lüst. I presume that you imagine a $2 \mathrm{G}$ solar field being extended radially all the way to the Earth, yielding $50 \mu \mathrm{G}$ by the inverse square law of flux conservation. But I thought Wilcox's work had shown that the field observed near the Earth is correlated with regions at the solar equator where the field is rather stronger than the $1 \mathrm{G}$ or $2 \mathrm{G}$ field at the solar poles. In that case the field near the Sun would have to fall off somewhat more rapidly than $1 / r^{2}$ - say, rather like $1 / r^{3}-$ going over into a $1 / r^{2}$ law further out. The point is important for the braking problem (especially for the stars with strong general magnetic fields). The torque on the star is quite sensitive to the detailed field structure.

Lüst: The $2 \mathrm{G}$ field is obtained when one uses the simple expression given in my Report [Equation (5), p. 254] that the radial component force is equal to $1 / r^{2}$ and takes $50 \mu \mathrm{G}$ near the Earth. The azimuthal angle at the distance of the Earth transcriber is then $135^{\circ}$.

Parker: You can add, that in general the gas pressure in the corona is capable of extending a field that is weaker than $2 \mathrm{G}$. Stronger fields resist expansion and you know that the magnetic field in space is from the weak field regions of the Sun.

Mestel: I agree that of the flux emanating from a local bipolar region with a strong field, only a part should be able to escape from the Sun and reach the neighborhood of the Earth; and I believe that Wilcox's observations confirm this. I would expect near the Sun both a dead zone, in which the field is strong enough to resist distortion, and a wind zone, with the flow near the Sun channelled along the quasi-dipolar fieldlines, but with the same lines further out too weak to resist being pulled into a roughly radial form by the wind. The relationship between the field strength seen at the Earth and that at the base of the same line as it emerges from the Sun will be more complicated than a simple $1 / r^{2}$ law.

Meyer: In one other respect the $2 \mathrm{G}$ extrapolation must be quite good. Though the flux tubes leading into the solar wind might have come from several restricted regions on the solar surface, as Mestel just mentioned, the flux must have smoothed out any irregularities by the time it has reached distances of the order of 10 to about $20 r_{\odot}$, and must have become very nearly radial everywhere. The reason is that at this distance all the multipole contributions of the inhomogeneous distribution on the surface have dropped off to quite small values. On the other hand the magnetic tensions are still the dominant dynamical feature. This requires that the magnetic field is in equilibrium with itself, that is, that the field is radial (except for the small 'garden hose' angle) and of equal strength on spheres around the Sun. Thus, though one only observes the magnetic flux at the orbit of the Earth, the $2 \mathrm{G}$ should also stand for all other directions.

Konyukov: I want to discuss the solar wind solution found by Parker and, in particular, the problem of heat flow. Essential to Parker's solution is the existence of a singular point, where the flow becomes supersonic. Under the assumption that the gas flow is stationary and spherically symmetrical I have expanded the heat flow 
function $W$ in a series with the inverse Reynolds number, $R_{e y}^{-1}$, as an expansion parameter. The expansion is valid only if energy dissipation is unimportant. In the zero-order approximation the heat flow is the same as in Parker's solution. However, for the coronal and interplanetary plasma $R_{e y}^{-1} \approx 1$ and the expansion is invalid, implying that Parker's solution is also invalid. [Condensed. (Ed.)] [Konyukov, M. V.: 1967, Geomagn. Aeronom. (Akad. Nauk SSSR) 7, 577.]

Lüst: In a recent review, Parker (1969) has discussed different models of the solar wind under different assumptions for the energy equation. (Parker, E. N.: 1969, Space Sci. Rev. 9, 325.)

Konyukov: I only wanted to mention that, if $R_{e y}^{-1} \approx 1$, we have no singular (sonic) point. I understand that magnetic fields and plasma turbulence decrease $R_{e y}^{-1}$, but these processes introduce other difficulties.

Thomas: Dr. Lüst, the energy input into the fast modes that you require is more than $10^{4} \mathrm{erg} \mathrm{cm}^{-2} \mathrm{sec}^{-1}$. What was the figure you and Biermann derived?

Luist: 1 remember a figure of about $10^{6} \mathrm{erg} \mathrm{cm}^{-2} \mathrm{sec}^{-1}$ for the chromosphere and the corona. We derived this number in two ways: (a) By estimating the production of acoustic noise at the upper part of the hydrogen convection zone. (This estimate is uncertain since it is difficult to calculate the efficiency of turbulence in generating acoustic noise); (b) By estimating the energy losses at the various levels. Both figures were in reasonable agreement and indicated about $10^{6} \mathrm{erg} \mathrm{cm}^{-2} \mathrm{sec}^{-1}$.

Thomas: You remember that at the preceding Gas Dynamics Symposium in Nice there was a big uncertainty about this figure. And now you are saying that 1 per cent of this flux will go out to $10 r_{\odot}$ and that you really need this! Well, all I will do is caution. The aerodynamic/hydrodynamic theory is very uncertain. Perhaps if we fix what is required to heat the chromosphere, actually the low corona, and if we fix the requirement at large distances from the Sun, we can perhaps tie down all that is needed. I would prefer such an estimate, because I do not believe any theoretical predictions on heat production and energy transport.

Tsytovich: Dr. Lüst, you say that the region in which the solar wind is observed is collisionless and that there are something like colliding beams. If these collisions are collective, what efficiency should you have for heating?

Luist: I would like to pass the buck* to Davis.

Davis: The temptation is to assume essentially 100 per cent efficiency over a sufficient distance. Near the Earth streams are observed with velocities ranging from $300 \mathrm{~km}$ $\mathrm{sec}^{-1}$ to $700 \mathrm{~km} \mathrm{sec}^{-1}$. These streams will interact and give all kinds of waves; the waves will damp, according to Barnes, and eventually, by the time you get to Jupiter, there are no streams left.

Baranov: Dr. Lüst, you mentioned that the temperature anisotropy of electrons is less than the temperature anisotropy of ions in the solar wind plasma. This is an experimental result obtained by Vela 4. How do you explain this effect?

* To pass the buck: Colloquial (American) expression meaning: to throw the responsibility to an other person. Buck also means a gymnastic apparatus for vaulting over. Here both interpretations seem possible. (Ed.) 
Liist: It can be explained by the mechanism proposed by Barnes. According to him the fast hydromagnetic waves would transfer energy to the protons, preferentially along the magnetic lines of force.

Parker: There is another effect, too, that the electrons have a large enough thermal conductivity to keep themselves warm at large distances from the Sun. But the protons have a very small thermal conductivity, and cannot keep themselves warm in the face of all the expansion. Lüst mentioned the opposite effect, i.e., warming the protons, rather than the electrons, by dissipation of hydromagnetic waves.

Davis: I would like to continue the discussion about the anisotropy of the electron and proton distribution functions in the solar wind. If you use only simple two-body collisions, they all should be much less isotropic than they are observed to be. It is clear that there are instabilities and collective modes which are maintaining the isotropy, to some extent, of both the electrons and the protons. The real issue is: Why are these collective modes more efficient for electrons? I suspect that any plasma physicist can give many reasons why the electrons cannot get a very anisotropic distribution.

Nikolaiev: I have a comment on Lüst's Report. Following earlier work (e.g., that of Parker) Korobeinikov and I studied the propagation in the solar wind of disturbances after a solar flare. Our model consisted of a strong point explosion in a gas, in which the density varies as $1 / r^{2}$ and the solar wind velocity is constant. We obtained an exact analytical solution. This non-similarity solution was compared with experimental data of the interplanetary plasma measured by two space probes simultaneously in different points of space. Résumé: (1) Only strong shock waves are rather well described by the similarity solutions. (2) If we include the motion of the solar wind, the calculated energy in a solar flare is decreased by a factor between 3 and 5. (3) The effective adiabatic exponent $\gamma$ can take both values $\frac{5}{3}$ (a perfect gas) and $\frac{2}{3}$ (one-dimensional gas flow). Gamma depends on the density and the velocity of the solar wind. (4) The propagation of a shock wave in a plasma with weak magnetic fields leads to formation of a magnetic trap for the solar protons.

I have also a question to Lüst. For the determination of the radius of the shock front between the solar wind and the interstellar medium we should include the subcosmic-ray pressure. The interstellar pressure, including the magnetic pressure $\left(\approx 10^{-11} \mathrm{erg} \mathrm{cm}{ }^{-3}\right)$ and the subcosmic-ray pressure $\left(\approx(3\right.$ to 4$\left.) \times 10^{-12} \mathrm{erg} \mathrm{cm}^{-3}\right)$, gives a radius of 20 or $30 \mathrm{AU}$ but not $200 \mathrm{AU}$.

Liist: The shock waves should be located at roughly $100 \mathrm{AU}$. But what pressure did you take? I used $10^{-12} \mathrm{dyne}^{-2}$ for the pressure of the interstellar medium. If you use your numbers, I agree with you that you have the shock wave closer to the Sun. As I said at the end of my talk, one might find the shock between 30 and 300 AU. But I think the radius of about 5 AU quoted earlier from Brandt seems to me too small.

Van Woerden: I think we do not know very well the local interstellar gas density. We might be in a cloud of 10 atom $\mathrm{cm}^{-3}$; we might be between clouds, so that the density is 0.2 atom $\mathrm{cm}^{-3}$.

Stecher: I disagree with Van Woerden. I think that the Ly- $\alpha$ measurements make it 
fairly clear that around the Sun the density within $100 \mathrm{pc}$ is quite low, between 0.1 and $0.2 \mathrm{~cm}^{-3}$.

Lüst: But, if you take such a low gas density, the gas pressure is unimportant compared to the other pressure components.

Baranov: I have a question for Dr. Lüst, which is connected to the problem of the interaction of the solar wind and the interstellar medium. The interstellar medium flows past the Sun with a velocity of $20 \mathrm{~km} \mathrm{sec}^{-1}$, which is a supersonic velocity. But the solar wind plasma is flowing with supersonic velocity too, and the velocity of the solar wind plasma is decreasing. Therefore we must have two shock waves; one in the interstellar plasma and one in the solar wind plasma. Could you comment on the distance between these shock fronts and the distance of these shock fronts from the Sun?

Lüst: You are certainly correct that one must take into account the motion of the solar system as a whole against the surrounding interstellar medium. This would cause a standing shock wave in front of the solar system (with respect to its apex motion). The distance of this shock wave from the boundary of the region where the magnetic field lines are still connected with the Sun may be estimated in a similar way as for the standing shock waves in front of the Earth's magnetosphere.

Davis: In the discussion this morning, Lüst said the solar system was surrounded by a sphere with a radius of the order of $1000 \mathrm{AU}$, filled up with old solar wind which had gone through the shock and was sitting there waiting to recombine. Is not this going to have some effect on the charge exchange with the possible incoming neutral particles he also mentioned? The neutrals in the interstellar gas have a chance to exchange charge in this region. The density is very low but the extent is very large. This may have a significant effect on the calculations.

Baranov: Dr. Lüst, Williams (1965) calculated the radius of the sphere ionized by solar radiation. He found a radius of about $1000 \mathrm{AU}$. You have a much lower value. Why?(Williams, R. E. : 1965, Astrophys. J. 142, 314.)

Luist: Williams assumed in his calculation for the extended HII region around the Sun that the solar system is at rest. In this way he obtained a radius of about 500 to $1000 \mathrm{AU}$. However, since the solar system is moving with a velocity of about $20 \mathrm{~km}$ $\mathrm{sec}^{-1}$, the neutral hydrogen atoms coming from a distance larger than $1000 \mathrm{AU}$ will penetrate into the $\mathrm{HII}$ region and will be ionized by charge exchange or by photoionization only at about 30 to $100 \mathrm{AU}$.

Parker: There is a basic question discussed earlier as to whether the interstellar medium some distance outside the solar system is in fact neutral or ionized. If it is neutral, one has the situation Lüst has described. But if it were ionized, there would be no possibility for charge exchange, and one has the interplay of two ionized gases.

Field: A general comment concerning the nature of the interstellar medium in the vicinity of the Sun. We heard from Stecher that Ly- $\alpha$ absorption lines in spectra of nearby stars indicate a density of about 0.1 to $0.2 \mathrm{~cm}^{-3}$. Is it possible that this medium is in fact the intercloud medium that has been discussed earlier in this Symposium? If so, its temperature is expected theoretically to be about $7000 \mathrm{~K}$. And if the medium 
were unaffected by the Sun, its pressure would be approximately $0.3 \times 10^{-12} \mathrm{erg} \mathrm{cm}^{-3}$. If, on the other hand, it is significantly ionized, either by the Sun or possibly by another nearby star, its pressure would be about twice that value. Some people have suggested that the intercloud medium is in fact fully ionized by perhaps quite distant early-type stars. In one paper it was suggested that in fact we live inside the Hil region of the Gum Nebula, which is believed to be ionized by $\zeta$ Puppis and $\gamma$ Velorum, two stars that Stecher mentioned (see p. 295). ${ }^{*}$ In that case we expect that the medium is in fact completely ionized out at least to several tens of parsecs from the Sun. That may be of some importance in calculating the interaction with the interstellar medium. Finally, a comment on the pressure of the surrounding magnetic field, which Lüst emphasized. Presumably, such a pressure is highly anisotropic, and consequently the shock itself may be nonspherical. Is there any possibility of testing that? The observations of Kurt and Sunyaev might throw light on this question.

Luist: I don't know of any observations, but I would agree that the shock would not be spherical.

Spiegel: Concerning anisotropy produced by a surrounding field, I worried about the same question re supernovae remnant observations. There was a paper specifically on this by Bernstein and Kulsrud (1965). Woltjer mentioned that they found a very small anisotropy of the shell for a supernova explosion in an ambient magnetic field. (Bernstein, I. B. and Kulsrud, R. M.: 1965, Astrophys. J. 142, 479.)

Parker: You can also do a stationary solution, and again the shock is very nearly spherical. Not quite, but deviations are small.

Meyer: There is another source of anisotropy in the solar wind post-shock region that might produce even larger effects. If the magnetic field remains frozen in to some degree, it will be substantially wound up in the equatorial plane but not in the polar direction. The resulting large 'magnetic pressure' gradients in latitude must have important dynamical effects on the flow direction.

\section{M. Gordon: On the Interpretation of Radar Studies of the Sun}

The origin of the solar wind is one of the most difficult problems of solar physics. The main obstacle for the solution of this problem is the absence of observational evidence on the physical conditions and the motions of the coronal plasma at distances $r$ from the center of the sun between 1.4 and $10 R_{\odot}$. It is between these distances that acceleration of the solar wind probably takes place. In principle, direct measurements of the velocities of coronal plasma can be carried out with the aid of the radar explorations of the Sun. This method appears promising because of the existence of an optimum frequency range extending from $40 \mathrm{MHz}$ down to lower frequencies. The range corresponds to reflection layers with $r \geqslant 1.4 R_{\odot}$ ( $r$ represents distance from the center of the Sun). However, the interpretation of the results of long standing radar

* Carruthers $(1968$, Astrophys. J. 151, 269) reports the presence of interstellar Ly- $\alpha$ absorption in both $\gamma$ Vel and $\zeta$ Pup, with hydrogen surface densities of $(3$ and 6$) \times 10^{19} \mathrm{~cm}^{-2}$, respectively. This excludes the possibility that the Sun is inside the Gum Nebula. (Ed.) 
experiments at $38 \mathrm{MHz}$ (James, 1964, 1966) is rather problematic because the almost symmetrical distribution of frequency shifts and the changes of effective cross sections observed are incompatible with the conventional theories of formation of reflected signals (Gordon, 1967, 1968a, b). Recently, it has been argued that the reflected signal may be formed by scattering of electromagnetic waves on turbulent pulsations in the coronal plasma (for this process see the Report by Kadomtsev and Tsytovich, p. 108). In the light of this new concept the radar investigations of the Sun form diagnostics of the coronal plasma on a large scale. The observed frequency displacements are no longer Doppler shifts, but result from combination scattering on ion acoustic waves (Gordon, 1968b, c) or from four-plasmon scattering on high frequency plasma waves as was supposed by Liperovski, Tsytovich, and myself. The major portion of the reflected signals is formed in the corona above the plages, while reflections from the quiet corona are weak or completely absent (Gordon, 1968c, 1969). Identification of the place of origin of the reflected signal enables us to define the velocity of the scattering layer by the average 'violet' shift. An analysis of the spectra of radar echoes from the Sun (James, 1964) reveals that a sometimes rapid rise takes place in the velocity of the solar wind in the corona above the plages. In different experiments the gradient of velocity changes over a quite wide range. From the scanty data published I reached the preliminary conclusion that higher effective cross sections (Gordon, 1969) are accompanied by steeper velocity gradients. In a further development of the theory of the solar wind (Bisnovatyi-Kogan and Gordon, 1969) we assumed that the rapid increase of velocity is caused by strong heating which leads to the acceleration of subsonic flow in a relatively thin layer. The correlation of both strong radar echoes and steep velocity gradients with the increase of level and fluctuations of the natural radio noise from the Sun suggests that the heating derives from coronal plasma turbulence excited by beams of accelerated electrons (Gordon, 1968a). From this model, we predict a rise of temperature up to $4 \times 10^{6}$ to $10^{7} \mathrm{~K}$. Velocities up to $120 \mathrm{~km} \mathrm{sec}^{-1}$ are reached at a relatively low level $r \approx 1.5 R_{\odot}$.

The next desirable step is the investigation of the structure of the layer, where the acceleration takes place, including transport phenomena. In this connection some important questions arise: How frequent are these accelerations of plasma above the plages? What is the duration of these events? Are they stationary? In addition to these a problem of great importance is to determine the correlation of these events with other characteristics of active regions and also with geophysical indices.

Two of the most important observational problems connected with the radar explorations of the Sun are the origin of weak scattered components and the variation of the structure of the reflected signal on a time scale of about $1 \mathrm{sec}$. The necessity of continuous radar patrolling of the Sun at different frequencies with adequate resolving power both in time and space can hardly be overestimated. Simultaneous observations of natural radio emission by the Sun at different frequencies may be of great value (Gordon, 1968a, b). The precise measurements of the coordinates of sources of various kinds of outburst, their small scale structure, motions, and frequency drifts (Wild, 1967; Philips, 1968) are very desirable. [Condensed. (Ed.)] 


\section{References [to Gordon's remark]}

Bisnovaty-Kogan, G. S. and Gordon, I. M.: 1969, Astron. Zh., in press.

Gordon, I. M.: 1967, Astron. Circ. Akad. Nauk SSSR, N447.

Gordon, I. M.: 1968a, Astron. Zh. 45, 1002.

Gordon, I. M.: 1968b, Astrophys. Lett. 2, 49.

Gordon, I. M.: 1968c, Astron. Circ. Akad. Nauk SSSR, N487.

Gordon, I. M.: 1969, Astrophys. Lett. 3, 181.

James, J. C.: 1964, IEEE Trans. Ant. Propag. AP-12, 876.

James, J. C.: 1966, Astrophys. J. 146, 356.

Philip, K. W.: 1968, Astron. J. 73, S197.

Wild, J. P.: 1967, Proc. Astron. Soc. Austr. 1, 59.

Van de Hulst: I think that it is quite clear that these radar measurements contain a lot of information. My main point of doubt is: do they contain relevant physical information or irrelevant geometrical information? The point being that if you have in the radar beam a perfectly reflecting sphere, large compared to the wavelength, then you have one reflection point in the middle, and the radar cross-section is equal to $\pi r_{s}^{2}$, where $r_{s}$ is the radius of the sphere, which in this case is equal to the $r_{c}$ radius of curvature at the center point. If, however, the sphere is buckled somewhat, $r_{c} \neq r_{s}$ at the reflection point and you will get many values of the cross section $\pi r_{c}^{2}$ which may be both larger or smaller than $\pi r_{s}^{2}$. Radial velocities may be explained in this way, if by a slight motion the main reflection shifts from a nearer to a farther point. My question is: Do you have any good criterion to discriminate against these geometrical effects?

Gordon: (1) The quiet corona is too poor a reflector to explain the very strong signals that occur and (2) there is a strong correlation between plage areas and reflective cross sections. [Answer condensed. (Ed.)]

Severnyi: Observations at the Crimea Observatory give predominantly downward motions, whereas you find an average upward motion.

Gordon: The downward motions mentioned by Severnyi are in the chromosphere. The radar signal cannot penetrate in such a low part of the solar atmosphere, but is reflected high in the corona. Somewhere in the corona the acceleration has to take place of plasma which initiates the solar wind. 